\title{
Micro machine tool oriented optimum design of 3-RPS parallel mechanism with large titling and uniform deflecting capacities
}

\author{
Gang ZHAO*, Yang DENG*, Wenlei XIAO* and Yazui LIU* \\ * School of Mechanical Engineering and Automation, Beihang University \\ 37 Xueyuan Road, Haidian District, Beijing, 100191, China \\ E-mail: xiaowenlei@buaa.edu.cn
}

Received: 2 November 2016; Revised: 7 July 2017; Accepted: 30 July 2017

\begin{abstract}
Parallel kinematic mechanism has shown its advantages as a high-precision motion stage in a machine tool. In this paper, a RPS-XY hybrid kinematic structure TRIPOD actuated by linear ultrasonic motors is proposed and designed for a five-axis ultra-precision micro machine tool. In the designing stage, both the titling capacity and the consistency of the deflecting abilities in all directions need to be carefully designed to overcome the platform's limitation in the freeform surface machining. This study proposes an optimizer that can automatically search for a set of design parameters of the 3-RPS parallel part to generate large titling and uniform deflecting capacities simultaneously. A specified orientation workspace is calculated to evaluate the ability of rotation. Additionally, a new index is introduced to justify the uniform deflecting capacity. By evaluating those factors as the objective functions, a modified NSGA-II algorithm is employed for the optimum design with the consideration of discretely selected linear guides and spherical joints from a predefined database, which contains the abstracted engineering models of commercial products. Optimal results verify the feasibility and effectiveness of the proposed optimizer in designing a physical platform. The technique is extensible to most of other parallel mechanisms as a standard procedure.
\end{abstract}

Key words : 3-RPS parallel mechanism, Micro machine tool, Five-axis machining, Rotational capacity, Multiobjective optimization

\section{Introduction}

Parallel Kinematic Mechanisms (PKMs) have been successfully used as high-precision motion stages in machine tools. Generally, they have the advantages of high rigidity, low inertia, and high dynamics, whereas they also suffer from small workspace and limited flexibility (Huda and Takeda, 2007) (Koenigsberger and Tlusty, 2013). Recently, many design criteria have been proposed to evaluate the PKMs' machining performance, such as workspace size, flexibility, etc. In order to balance those requests, the hybrid cooperating kinematics structure (HCKS) is frequently adopted in the application of five-axis machining ( $\mathrm{Li}$ et al., 2007) (Shneor and Portman, 2010). However, its application in a micro machine tool is challenged due to the size limitation of components. Firstly, people usually wish to have a relatively large tilting angle for more freedoms in freeform machining, while the motion ranges of key components like linear guides and spherical joints are limited compared to their structural sizes. Secondly, in case of a limited orientation capability, only the minimum tilt angle (Shi et al., 2012) is not suffice to justify the freeform machining performance of a micro machine tool, as it is not able to represent the deflecting abilities in all directions (Wang et al., 2001). Actually, a large uniform deflecting capacity cannot only fasten the rapid motions, but also facilitate setup changes and reduce the machining leadtime (Hong and Kim, 2000). Thirdly, the critical components used in a micro machine tool are usually with high precision and great costs, thus their discrete structural parameters need to be carefully selected in the design, so as to make the most correct choice and avoid the risk of waste. In order to overcome the above difficulties, this paper develops an optimum design algorithm that can automatically search for a set of optimal parameters to design the parallel mechanism in a micro machine tool. 
In the last decades, amounts of researches have been implemented to optimize the rotational capacity in designing a PKM, in which the orientation workspace size and shape are the most frequently discussed and evaluated criteria. Kim et al. studied the machine tool workspace characteristics of the conventional Stewart-Gough platform with the maximum tilting angles $46^{\circ}$ (ball joint range $55^{\circ}$ ) (Kim et al., 2000). Li et al. proposed an optimized 3-RPS parallel A3 tool head with the maximum nutation $40^{\circ}$, the spherical joint of which is a combination of three revolute joints (Li et al., 2010). Liu et al. developed 3-DOF HANA, a spatial PKM using the kinematic chains of one PRU (the universal joint is substituted as two revolute joints) and two PRPaR, and achieving high tilting capacity ( $\pm 44.82^{\circ}$ ) (Liu et al., 2005). Actually, the ranges of the tilting angles are limited by the corresponding critical joint capacities. Most of the above optimizations only involve the maximum tilt capability, hence may result in non-regular shape of the orientation workspace. In the study by Shi et al. (2012), standard deviation was introduced to describe the oscillation between the maximum and minimum tilt angle, and the oscillation indicates the regularity of the shape of the workspace (defined as a circle). Wang et al. introduced a variable named the smallest reachable nutation angle to describe the conic face constraint of the workpiece orientation (Wang et al., 2001). Nonetheless, it is still hard to characterize the shape of the orientation workspace by those criteria. Furthermore, the structural parameters of a PKM stage are usually continuously derived, which may not fit the micro machine tool, as the structural parameters of some components behave discretely.

This paper proposes the design process of a 3-RPS stage (Hunt, 1983) used in a micro machine tool. An optimization algorithm is developed to fasten and optimize the design. Accordingly, this paper represents the orientation workspace in a new way, and a uniform deflection factor is proposed to quantify the deflecting consistency of the stage in all directions. Furthermore, design parameters of the stage are classified into two categories according to their engineering significance, based on which the effects of them on the rotational capacity are analyzed. Finally, by using a modified NSGA-II algorithm capable of multi-objective optimization with mixed-discrete variables, the optimization is realized for large rotational capacity of 3-RPS PKM. With considering other important performance indexes, the optimal results can be conducted into a physical stage.

\section{Analysis of the orientation workspace}

Figure 1 shows the concept of a micro machine tool, consisting of an isolation platform, a marble gantry holding an ultra-high speed spindle, and a five-axis high-precision TRIPOD stage. The TRIPOD stage is hybridized with a XY base (enhances the Cartesian workspace) and a 3-RPS stage (provides the rotation capability). In case of five-axis freeform machining, the rotation capability is vitally important, and in the proposed micro machine tool it is mainly limited by the 3-RPS stage. Therefore, the rotation workspace of it needs to be analyzed for the consideration of optimization. This section introduces the calculation of the orientation workspace for the 3-RPS stage. The inverse kinematics is introduced from the work by Lee and Shah (1988) and derived as follows:

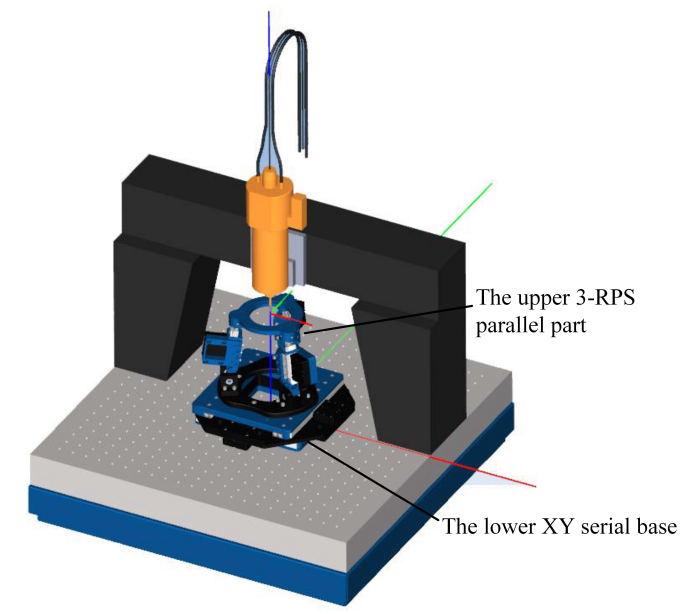

Fig. 1: Micro machine tool

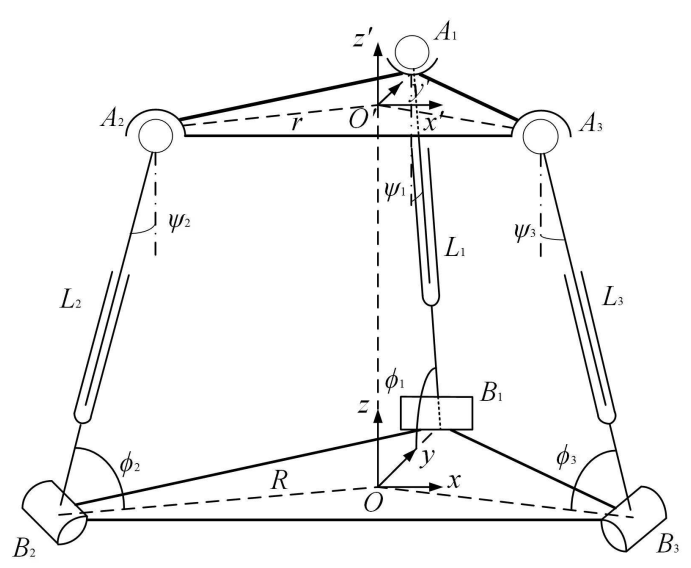

Fig. 2: Schematic of 3-RPS parallel part

The schematic of the 3-RPS stage is illustrated in Fig. 2, where $o x y z$ denotes the base frame, and $o^{\prime} x^{\prime} y^{\prime} z^{\prime}$ represents the move frame with respect to the base. Suppose the frame $o^{\prime} x^{\prime} y^{\prime} z^{\prime}$ is presented by $\left(x_{c}, y_{c}, z_{c}, \alpha, \beta, \gamma\right)^{T}$, in which the Euler angles obey the $z-y-z$ order. Wherein, the variables $\alpha, \beta, z_{c}$ is determined independently by the 3-RPS stage. 
Correspondingly, the link lengths are calculated by:

$$
\begin{aligned}
L_{1}^{2}= & R^{2}+r^{2}+x_{c}^{2}+y_{c}^{2}+z_{c}^{2}-2 R y_{c}+2 r\left(C_{\alpha}^{2}+C_{\beta} S_{\alpha}^{2}\right)\left(y_{c}-R\right)+r\left(C_{\beta}-1\right) S_{2 \alpha} x_{c}-2 r S_{\alpha} S_{\beta} z_{c} \\
L_{2}^{2}= & R^{2}+r^{2}+x_{c}^{2}+y_{c}^{2}+z_{c}^{2}+R y_{c}+\sqrt{3} R x_{c}-r\left[S_{\alpha} C_{\alpha}\left(C_{\beta}-1\right)+\sqrt{3}\left(C_{\alpha}^{2} C_{\beta}+S_{\alpha}^{2}\right)\right]\left(x_{c}+\frac{\sqrt{3}}{2} R\right) \\
& +r\left[C_{\alpha}^{2}+C_{\beta} S_{\alpha}^{2}+\sqrt{3} C_{\alpha} S_{\alpha}\left(C_{\beta}-1\right)\right]\left(y_{c}+\frac{1}{2} R\right)+r S_{\beta}\left(S_{\alpha}+\sqrt{3} C_{\alpha}\right) z_{c} \\
L_{3}^{2}= & R^{2}+r^{2}+x_{c}^{2}+y_{c}^{2}+z_{c}^{2}+R y_{c}-\sqrt{3} R x_{c}-r\left[S_{\alpha} C_{\alpha}\left(C_{\beta}-1\right)-\sqrt{3}\left(C_{\alpha}^{2} C_{\beta}+S_{\alpha}^{2}\right)\right]\left(x_{c}-\frac{\sqrt{3}}{2} R\right) \\
& -r\left[C_{\alpha}^{2}+C_{\beta} S_{\alpha}^{2}-\sqrt{3} C_{\alpha} S_{\alpha}\left(C_{\beta}-1\right)\right]\left(y_{c}+\frac{1}{2} R\right)+r S_{\beta}\left(S_{\alpha}-\sqrt{3} C_{\alpha}\right) z_{c}
\end{aligned}
$$

where $S_{\alpha}=\sin \alpha, C_{\alpha}=\cos \alpha, S_{\beta}=\sin \beta, C_{\beta}=\cos \beta$ and $S_{2 \alpha}=\sin 2 \alpha$.

\subsection{Orientation workspace}

The orientation workspace is defined in a cylindrical coordinate system, which maps $\left(\alpha, \beta, z_{c}\right)$ to $(\rho, \varphi, z)$, and its mapping relationship is $\rho=\sin \beta, \varphi=\alpha$ and $z=z_{c}$.

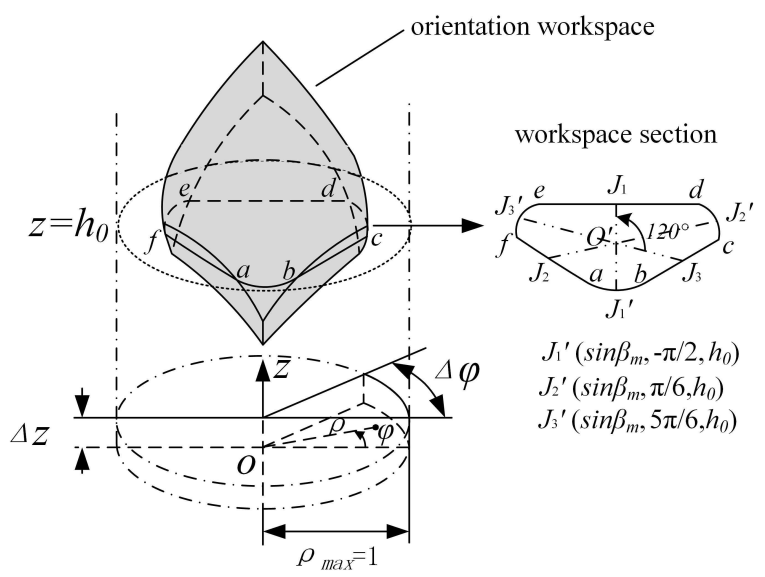

Fig. 3: Orientation workspace definition

As is shown in Fig. 3, the shape of the orientation workspace forms a quasi-hexahedron. Therefore, the plane at height $z=h_{0}$ intersects with the workspace and produces a shape as $a \rightarrow b \rightarrow c \rightarrow d \rightarrow e \rightarrow f$. Due to the inplane constraints from the revolute joints, the cylindrical coordinates of the three spherical joints are in fixed directions of angles $\varphi_{1}=-5 \pi / 6, \varphi_{2}=-\pi / 6$ and $\varphi_{3}=\pi / 2$. Supposing $O^{\prime}$ is the center of the section, the radials in the directions of $\varphi_{i}(i=1,2,3)$ intersect with the boundary at $J_{i}(i=1,2,3)$, and the extensions of $J_{i} O^{\prime}(i=1,2,3)$ intersect with the boundary at $J_{i}^{\prime}(i=1,2,3)$. Obviously, $J_{i} J_{i}^{\prime}(i=1,2,3)$ are the three symmetric lines of the section.

According to the previous definitions, the orientation workspace can be obtained by summarizing the intersection planes at different heights $z$. Hence, an iterative algorithm has to be developed to numerically calculate the intersection shapes by sampling the boundary coordinates in each direction. The searching steps are denoted as $\Delta z$ and $\Delta \varphi$ respectively. In addition, the computation costs can be significantly reduced by using the symmetry of the workspace, as only one-sixth of the intersection plane needs to be computed (Fig. 3).

Moreover, the calculation of boundary coordinates is the most computing consumed process, thus the bisection algorithm is employed to obtain a better efficiency:

1) Suppose in a direction of angle $\varphi$, the interval within which to conduct the bisection process is set to [0,1]. Thus, the iterative process begins by setting $\rho_{(1)}$ to the mid-point of the interval, i.e., $\rho_{(1)}=0.5$.

2) By using the inverse kinematics, the lengths of links $L_{i}^{(1)}(i=1,2,3)$ and the angles of swing of spherical joints $\psi_{i}^{(1)}(i=1,2,3)$ can be calculated. If $L_{\min } \leq L_{i}^{(1)} \leq L_{\max }$ and $-\psi_{\max } \leq \psi_{i}^{(1)} \leq \psi_{\max }$, then $\rho_{(2)}$ is chosen to be the mid-point of the interval $\left[\rho_{(1)}, 1\right]$. Otherwise, $\rho_{(2)}$ is chosen to be the mid-point of the interval $\left[0, \rho_{(1)}\right]$.

3) The above process is repeated until $\left|\rho_{(k)}-\rho_{(k-1)}\right|<\varepsilon_{\rho}$, hence the final value $\rho_{(k)}$ indicates the boundary in this direction. 
This section proposes an exemplary 3-RPS stage, whose structural parameters are $R=70 \mathrm{~mm}, r=55 \mathrm{~mm}, \psi_{\max }=30^{\circ}$ and $L_{i} \in[95 \mathrm{~mm}, 120 \mathrm{~mm}](i=1,2,3)$.

Figure 4 demonstrates the computed workspace with $\Delta \varphi=1^{o}, \Delta z=\left(z_{\max }-z_{\min }\right) / 100$ and $\varepsilon_{\rho}=10^{-5}$.

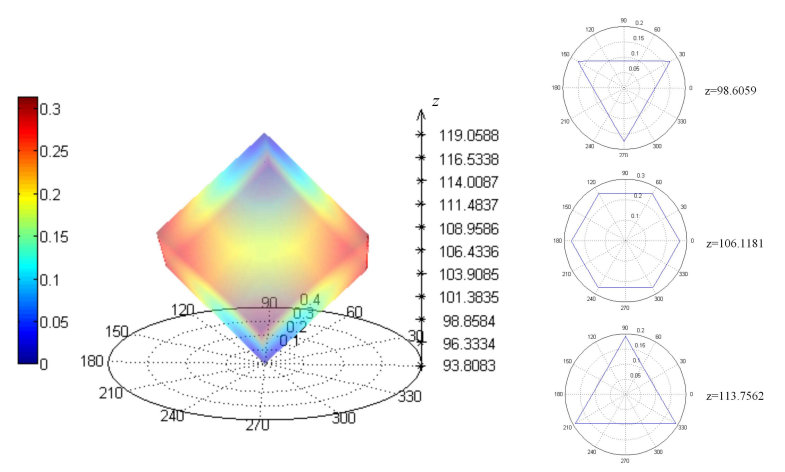

(a) Orientation workspace and specified workspace sections
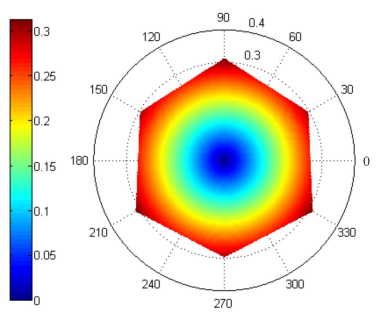

(b) Projection onto $O \rho \varphi$ plane

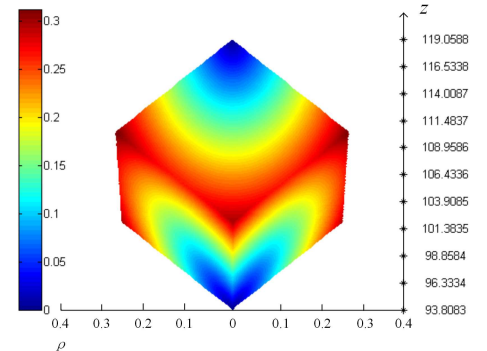

(c) Projection onto $O \rho z$ plane

Fig. 4: Orientation workspace specification

\subsection{Uniform deflection factor}

Generally, the workspace volume is a critical index in most optimization studies. However, a large workspace might have a long but narrow shape, thus is not suitable for freeform surface machining due to its poor rotational capacity. In this point of view, the maximum tilting angle and the deflecting consistency of in all directions should be considered as the most important criteria.

In this paper, a uniform deflection factor is introduced to quantify the deflecting consistency in all directions. This factor is defined as the proportion of the maximum inscribed workspace (formed by summarizing the maximum inscribed circles of the workspace sections at different heights) in the whole workspace, and it can be obtained as a volume ratio

$$
\delta=V_{I} / V
$$

where $V_{I}$ and $V$ denote the volumes of the maximum inscribed workspace and the whole workspace, respectively. Obviously, a large uniform deflection factor is preferred for complex surface manufacturing in five-axis NC machining. When the stage reaches a point $P$ in the Cartesian workspace, a conic face of the minimum tilting angle (denoted by $\beta_{M}$ ) is obtained to constrain the maximum deflection of the stage consistent in all directions, as shown in Fig. 5. And the base of the cone is shaped as the maximum inscribed circle at the height of the point $P$. Thus, usually the maximum inscribed workspace is in the shape of a paraboloid of revolution around the $\mathrm{z}$ axis.

The calculation of the volume of the workspace $V$ is given as below:

$$
\begin{aligned}
& \Delta V_{j}=\frac{\left(A_{j}+A_{j+1}\right) \Delta z}{2}(j=1,2, \ldots, N-1) \\
& V=\sum_{j=1}^{N-1} \Delta V_{j}
\end{aligned}
$$




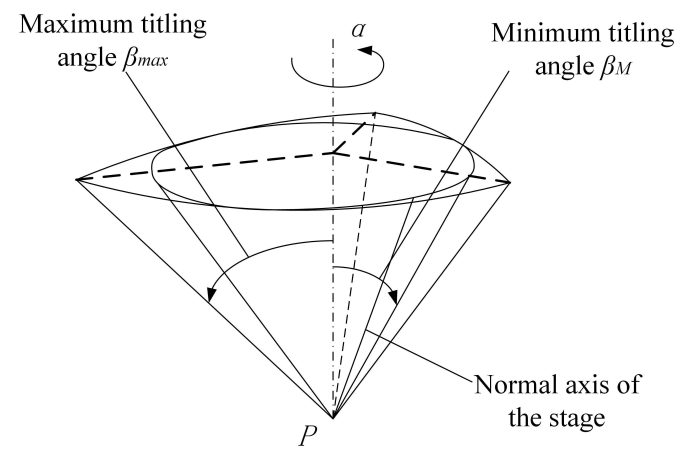

Fig. 5: Schematic of the uniform deflecting of the stage

where $N$ is the number of iterations in the $z$ axis, and $A_{j}$ is the area of the $j^{\text {th }}$ workspace section obtained by

$$
A_{j}=\sum_{s=1}^{K-1} \frac{\left(\rho_{s}+\rho_{s+1}\right) \Delta \varphi}{4}(s=1,2, \ldots, K-1)
$$

in which $K$ is the number of iterations in the direction, and $\rho_{s}$ denotes the boundary in the $s^{\text {th }}$ direction.

Similarly, the volume of the maximum inscribed worksp-ace $V_{I}$ can also be calculated. And, the uniform deflection factor can be determined by Eq. (4).

\section{Continuous and discrete parameters}

The engineering assembly model of 3-RPS stage is given in Fig. 6, whose structural and dimensional parameters are given in Fig. 7, Fig. 8, and Fig. 9.

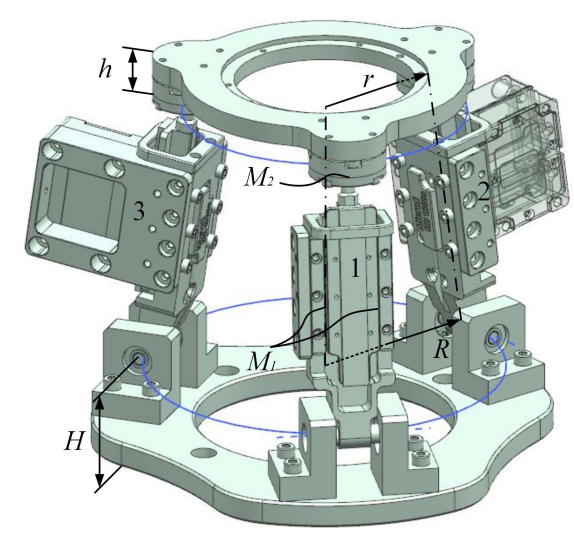

Fig. 6: Engineering assembly model of 3-RPS parallel mechanism

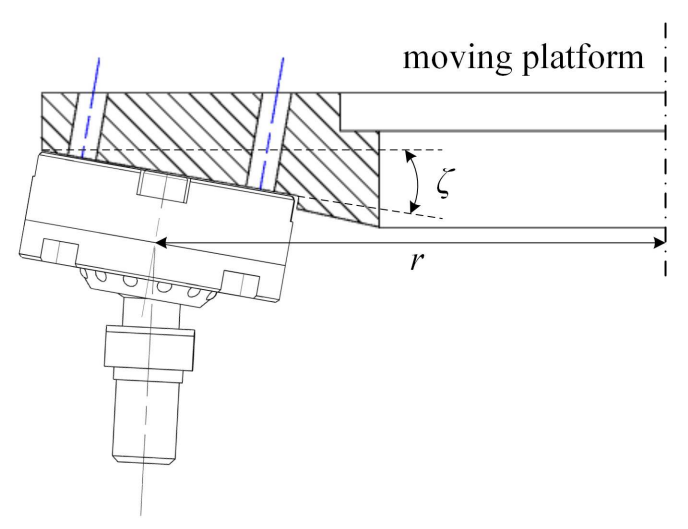

Fig. 7: Mounting configuration of the spherical joint

\subsection{Engineering definition of parameters}

In the engineering optimum design, critical parameters need to be considered and redefined according to their engineering significance. These parameters can be categorized as continuous and discrete. The continuous parameters are structural variables that can be continuously customized, which include the radii of the moving and base platforms $(r, R)$, the heights of the moving and base platforms $(h, H)$ (Fig. 6), the mounting angle of the spherical joint $\zeta$ (Fig. 7), and the customizable lengths of the link $S_{1}, S_{2}, S_{3}$ (Fig. 9(a)). The discrete parameters are derived from the standard commercialized components (i.e., the linear guide $M_{1}$ and the spherical joint $M_{2}$, as shown in Fig. 6) and thus can only be optimized as discrete variables. In this study, the cross roller guide model (VR series) from THK and the spherical rolling joint (SRJ series) from HEPHAIST SEIKO are chosen. Accordingly, the discrete parameters include the original rail length $L_{0}$, the stopper sickness $T$, the maximum stroke $P$ (Fig. 8(a)), and the permissible angle of swing $\psi_{\max }$, the length of axis of 


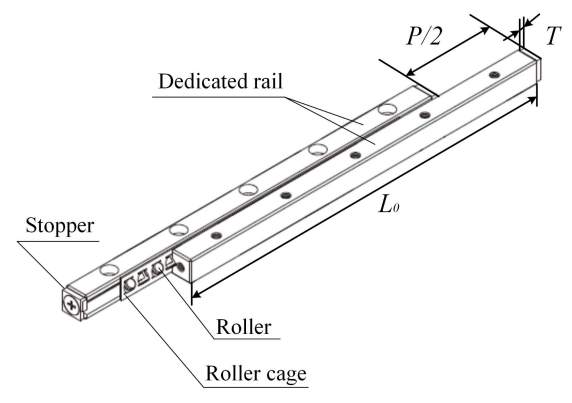

Cross Roller Guide Model VR

(a) Linear guide specification

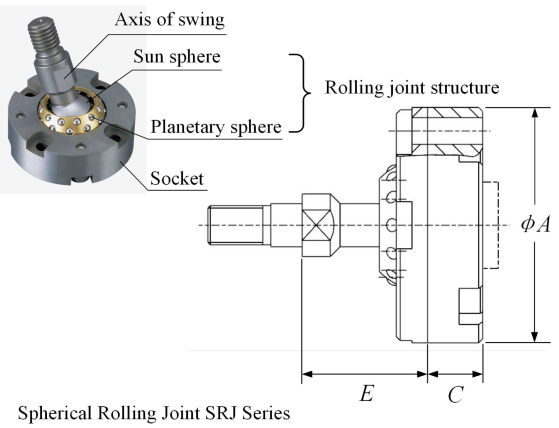

(b) Spherical joint specification

Fig. 8: Commercialized components specification

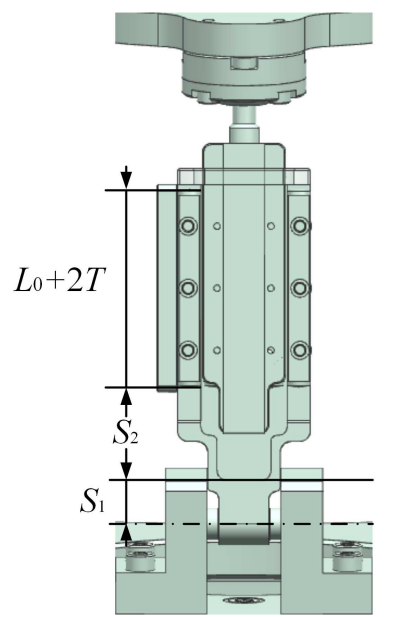

(a) Model of the link

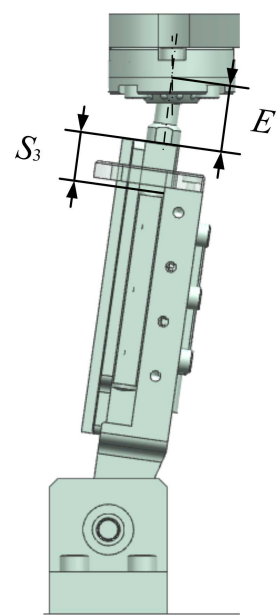

Fig. 9: Link parameters specification

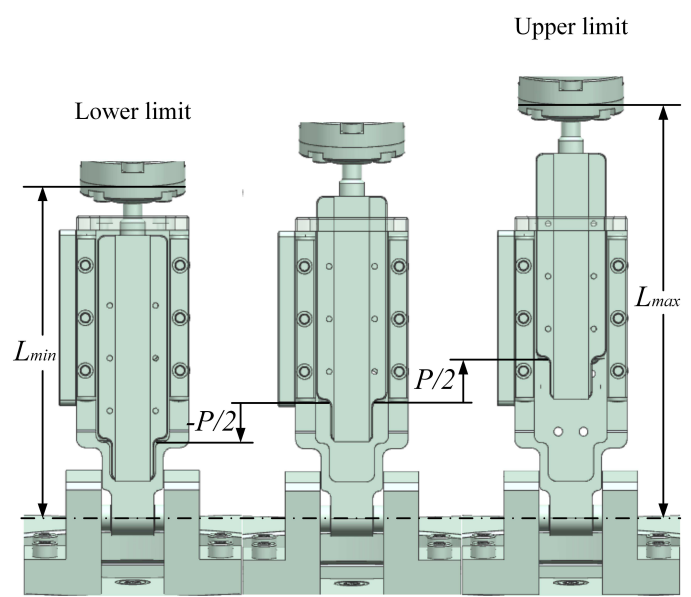

(b) Lower and upper limits of the link

swing $E$ (Fig. 8(b)). Totally sixteen products of the VR series and three products of the SRJ series can be retrieved from the database in Appendix A.

The engineering model of the link is shown in Fig. 9(a), in which the linear guides are at zero stroke and the corresponding length of the link is

$$
L_{z s}=\left(S_{1}+S_{2}+S_{3}\right)+\left(L_{0}+2 T\right)+E
$$

where the first part is an addition of continuous parameters (denoted by $S$ ) while the second and the third parts involve discrete parameters. Moreover, the lower and upper limits of the link are obtained when the linear guides reach $-P / 2$ and $P / 2$ respectively (Fig. $9(\mathrm{~b}))$.

$$
L_{\text {min }}=L_{z s}-P / 2 ; L_{\max }=L_{z s}+P / 2
$$

\subsection{Analysis of effects on rotational capacity}

In order to determine the decision variables of the optimization, the effects of both continuous and discrete parameters on the rotational capacity need to be analyzed.

(1) Radii of the moving and base platforms

By defining a radius ratio $\lambda=r / R$, the relation between the maximum tilting angle and the height of the stage in different radius ratios is shown in Fig. 10.

Figure 11(a) shows that both the maximum tilting angle $\beta_{\max }$ and the minimum tilting angle $\beta_{M}$ vary with $\lambda$, and Fig. 11(b) indicates the change of the uniform deflection factor $\delta$ with respect to $\lambda$. It is shown that $\beta_{\max }$ and $\beta_{M}$ increase 


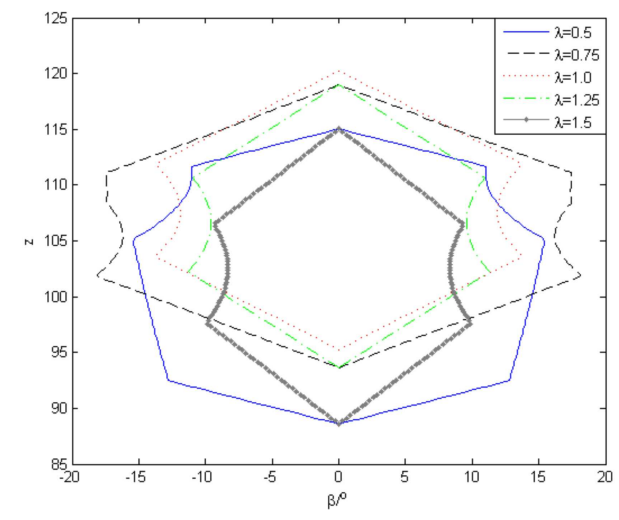

Fig. 10: Maximum tilting angles in different radius ratios

firstly then decrease with the increase of $\lambda$ and attend their extremes simultaneously when $\lambda=0.7$. And, $\delta$ is almost constant as $\lambda$ ranges from 0.8 to 1.6 .

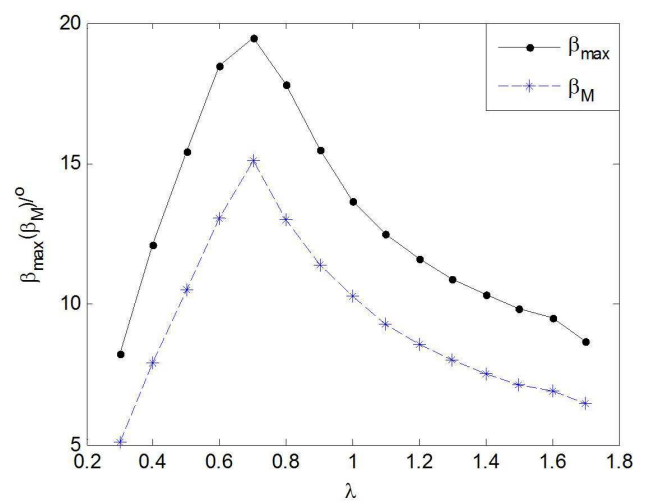

(a) Maximum and minimum tilting angles in different radius ratios

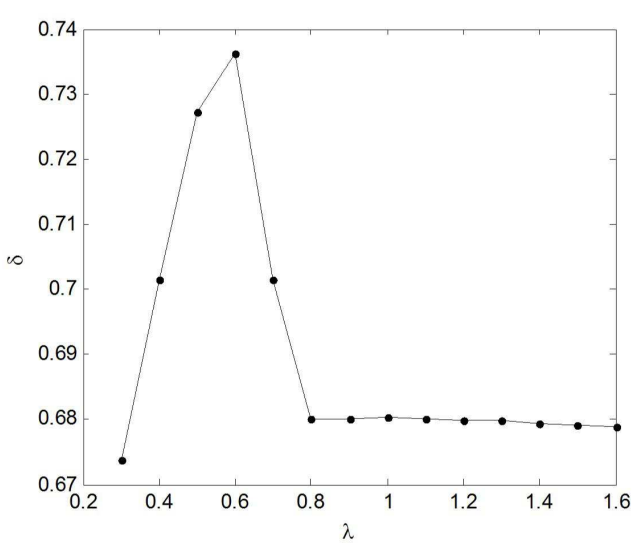

(b) Uniform deflection factors in different radius ratios

Fig. 11: Effects of radius ratio on rotational capacity

(2) Heights of the moving and base platforms

With the increase of $h$ and $H$, the points in the workspace are lifted in the positive $z$ axis. However, the lifting mechanism by $h$ is different from that by $H$, for the latter one is an equal-distance lifting for the coordinates of all the points while the former one (Fig. 12) is not.

As shown in the figure, the center of the section possesses the lifting distance of $h\left(P_{0} \rightarrow P_{0}^{\prime}, \Delta z_{0}=h\right)$, and a larger nutation angle $\beta$ means a smaller lifting distance $\left(P_{2}\right.$ versus $\left.P_{1}, \Delta z_{0} \cos \beta_{2}<\Delta z_{0} \cos \beta_{1}\right)$. Obviously, the mechanism does not change the maximum tilting angle. Furthermore, the volume of the sub-workspace formed by the $j^{\text {th }}$ workspace section $V_{j}^{\prime}$ can be calculated by double integrating in the polar coordinates

$$
\Delta V_{j}^{\prime}=\iint \frac{\rho d \rho d \varphi}{\cos \beta} \cdot \Delta z \cos \beta=\Delta z \iint \rho d \rho d \varphi=\Delta V_{j}
$$

It is suggested that the sub-workspace is with its original volume $V_{j}$, and the volume of the whole workspace stays the same. Thus, the mechanism does not change the uniform deflection factor either. In conclusion, both $h$ and $H$ have no effects on the rotational capacity.

(3) Mounting angle of the spherical joint

Obviously, the rotation of the link about the spherical joint is constrained by the mounting angle of the joint and determined by the workspace. In this sense, the effect of the mounting angle on workspace can be analyzed in two steps: 


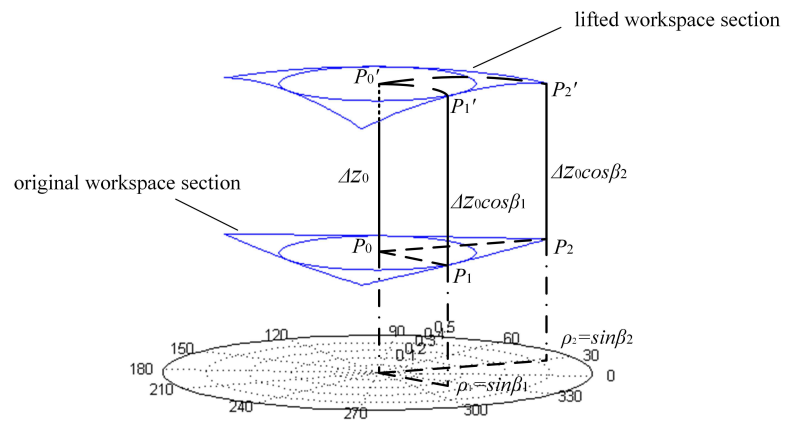

Fig. 12: Lifting mechanism of workspace by the height of the moving platform

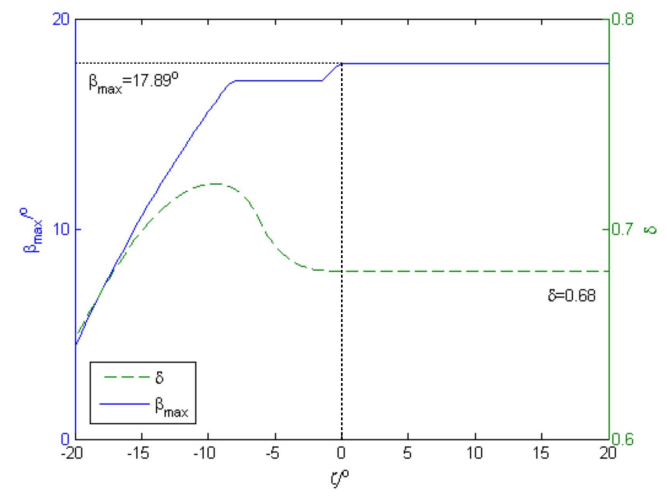

Fig. 13: Maximum tilting angles/uniform deflection factors in different mounting angles

calculate the motion range of the rotation angle of the link (denoted as $\left[\psi_{\text {Inf }}, \psi_{\text {Sup }}\right]$ )and then estimate the utilization degree of the rotation capacity of the joint.

Here we assume that the angle of rotation is an algebraic variable, whose positive direction is defined as the rotating direction close to the origin of the base platform. And its zero value is reached when the stage is at an original position of $L_{1}=L_{2}=L_{3}=L_{\min }$. In order to conduct the estimating process of the two-step analysis above, it is necessary to ensure that the interval $\left[\psi_{\text {Inf }}, \psi_{S \text { Sup }}\right]$ is a subset of the permissible angle range for the joint's swing $\left[-\psi_{\max }, \psi_{\max }\right]$, i.e., they share the same zero value. Thus the original position should also be the position where the link is vertical to the socket of the joint, and the corresponding mounting angle is given by

$$
\zeta_{0}=\operatorname{sign}(R-r) \arccos \left(z_{\min } / L_{\min }\right)
$$

Set the mounting angle to $\zeta_{0}$ and $\left[\psi_{\text {Inf }}, \psi_{\text {Sup }}\right]$ can be calculated. Then, the estimating process is given as below:

1) If $\psi_{\text {inf }}=-\psi_{\text {max }}$ and $\psi_{\text {sup }}=\psi_{\text {max }}$, the spherical joint reaches full utilization of its rotation capacity. Any variation of $\zeta$ leads to the workspace deformation;

2) If $\psi_{\text {inf }}>-\psi_{\max }$ and $\psi_{\text {sup }}<\psi_{\max }$, the spherical joint reaches part of utilization of its rotation capacity. There exists a range $\left[\zeta_{m}, \zeta_{M}\right]\left(\zeta_{m}<\zeta_{0}<\zeta_{M}\right)$, in which a variation of $\zeta$ has no effect on the workspace;

3) If $\psi_{\text {inf }}=-\psi_{\max }$ and $\psi_{\text {sup }}<\psi_{\max }$, the spherical joint reaches full utilization of its rotation capacity in the negative direction but part in the positive. There exists a range $\left[\zeta_{m^{\prime}}, \zeta_{M^{\prime}}\right]\left(\zeta_{m^{\prime}} \geq \zeta_{0}\right)$, in which a variation of $\zeta$ has no effect on the workspace;

4) If $\psi_{\text {inf }}>-\psi_{\max }$ and $\psi_{\text {sup }}=\psi_{\max }$, similarly the range free of effect is $\left[\zeta_{m^{\prime \prime}}, \zeta_{M^{\prime \prime}}\right]\left(\zeta_{M^{\prime \prime}} \leq \zeta_{0}\right)$.

In summary, a variation of the mounting angle within certain range results in the workspace deformation. For the exemplary architecture, $\zeta_{0}=9.1^{\circ}$ and motion range of the angle of rotation of the link is $\left[-17.2^{\circ}, 19.1^{\circ}\right]$. Fig. 13 shows the changes of $\beta_{\max }$ and $\delta$ with $\zeta$ in the range of $\left[-20^{\circ}, 20^{\circ}\right]$ (step size of $\zeta$ is $0.5^{\circ}$ ). Obviously, the range free of effect is $\left[0,20^{\circ}\right]$ with $\beta_{\max } \equiv 17.89^{\circ}$ and $\delta \equiv 0.68$.

(4) Other parameters

Other parameters include the discrete parameters $L_{0}, T, P, E$, and $\psi_{\max }$. Obviously, each of them has an effect on the rotational capacity.

\section{Optimization and implementation}

In this section, the 3-RPS parallel part in TRIPOD is to be optimized for large tilting capacity and good uniform deflecting capacity. Actually, the commercialized high-precision spherical joint is often with small permissible range of swing angle (within $\pm 30^{\circ}$ ), which makes the rotational capacity optimization is necessary for a micro machine tool.

\subsection{Genetic algorithms}

Genetic algorithms (GAs) (Whitley, 1994) are widely applicable stochastic searches and optimization methods. This kind of technique has been shown to solve nonlinear problems with good global optimization performance and robustness. In the studied case, there are both continuous and discrete parameters and the complicated workspace computation. Thus it 
is very hard to figure out the analytical expressions of the objective functions. Moreover, for other optimization techniques, only a few geometric parameters (Gosselin and Guillot, 1991) can be handled for the lack of convergence in such complex problems. Hence, the genetic algorithm is the best candidate.

To obtain the non-dominated solutions of the current case, the NSGA-II algorithm proposed by Deb et al. (2002) which is the most preferred algorithm to solve the multi-objective problems based on the principles of GA, is adopted.

\subsection{Decision variables}

The radius ratio $\lambda$, the customizable length of the link $S$, the mounting angle of the spherical joint $\zeta$, the discrete parameters $\left(L_{0}, T, P\right)$ from the linear guide and $\left(E, \psi_{\max }\right)$ from the spherical joint, are chosen as the decision variables. Non-negative integers representing the products of $M_{1}$ and $M_{2}$, is used to substitute $\left(L_{0}, T, P\right)$ and $\left(E, \psi_{\max }\right)$ as the decision variables. The vector of decision variables is

$$
x=\left(\lambda, S, \zeta, M_{1}, M_{2}\right)^{T}
$$

and their bounds or values are shown as below

Table 1: Bounds or values of the decision variables

\begin{tabular}{lllll}
\hline$\lambda$ & $S(\mathrm{~mm})$ & $\zeta\left(^{\circ}\right)$ & $M_{1}$ & $M_{2}$ \\
\hline $0.5 \sim 1.5$ & $10 \sim 200$ & $-30 \sim 30$ & $0,1, \ldots, 15$ & $0,1,2$ \\
\hline
\end{tabular}

\subsection{GA parameters setup}

The standard NSGA-II algorithm was designed for continuous variables without constraints, thus is not suitable for the current case and cannot be directly used. The parameters and corresponding modifications are:

Chromosome representation: Real number representation in the standard algorithm cannot support the coding of the discrete variables, while binary representation and Gray code representation can. Moreover, Gray coding improves the search efficiency compared with binary coding. Thus, Gary code representation is employed with the coding precision set to $10^{-4} \mathrm{~mm}(\mathrm{rad})$;

Selection function: Tournament selection process in the standard algorithm is preserved;

Genetic operators: Employ fixed-probability uniform crossover with $p_{c}=0.9$ and simple mutation with $p_{m}=0.1$ as the genetic operators for a high efficiency in processing the Gray-coded chromosome;

Population size: Population size is set to 20;

Evaluation function: Each evaluation function consists of two parts, an objective function modeled by a analytic hierarchy process (Chang, 1996), and an adaptive penalty function.

In the modeling of the objective functions, two secondary criteria are considered: the radius ratio $\lambda$ to ensure that the worktable should not be too small, and the motion range of the revolute joint (denoted by $\Delta \phi$ ) to ensure its compatibility with the flexible hinge. And compared with them, both the two major indexes $\beta_{\max }$ and $\delta$ are on the level of apparently significant, based on which the weight vector is figured out as $(0.9623,0.1925,0.1925)^{T}$. Thus, the objective functions are

$$
\begin{aligned}
& f_{1}(x)=0.9623 f_{01}(x)+0.1925 g_{1}(x)+0.1925 g_{2}(x) \\
& f_{2}(x)=0.9623 f_{02}(x)+0.1925 g_{1}(x)+0.1925 g_{2}(x)
\end{aligned}
$$

in which $f_{01}(x)=1-\beta_{\max }$ and $f_{02}(x)=1-\delta . g_{1}(x)$ and $g_{2}(x)$ are $\lambda$ related and $\Delta \phi$ related functions, respectively.

$$
\Delta \phi=\operatorname{acrcos}\left(|R-r| / L_{\max }\right)-\arccos \left(|R-r| / L_{\min }\right)
$$

The two evaluation functions are (Liu and Zhou, 2006)

$$
f_{p i}(x)=f_{i}(x)+C_{i}(x) \cdot p(x)(i=1,2)
$$

where the coefficient $C_{i}(x)$ is given by

$$
C_{i}(x)=1+\left|f_{i}(x)\right| /[1+p(x)](i=1,2)
$$


In conclusion, the optimization is formulated as:

$$
\min f_{p i}(x)(i=1,2)
$$

subject to:

- $R=100,0.5 \leq \lambda \leq 1.5,10 \leq S \leq 200,-30^{\circ} \leq \zeta \leq 30^{\circ}$;

- $M_{1}=\{0,1,2, \ldots, 15\}, M_{2}=\{0,1,2\}$;

- $\quad L_{\text {min }} \geq \max \{R, r\}, L_{\text {max }} \leq 2.5 \max \{R, r\}, S \geq P+S_{\text {Inf }}$.

where the constraints are established to ensure a reasonable mechanism dimension and avoid components interference.

\subsection{Results}

With the number of iterations set to 100, the Pareto front is shown in Fig. 14, and some non-dominated solutions with their criteria are given in Table 2. These solutions in decision space are listed in Table 3, which corresponds to some sets of optimal parameters. Surely there is no single solution for which all criteria are optimal simultaneously, and each solution is important in terms of some trade-off relationship among the criteria. Further, with considering other two performance indexes: the transmission index and the global condition index, an intermediate solution is selected. However, the part is not included here on account of the space limitation. The physical platform, which has large rotational capacity $\left(\beta_{\max } \approx 30^{\circ}\right.$ and $\left.\delta=0.80\right)$, is at its maximum tilt as shown in Fig. 15(a). The prototype of micro machine tool is presented in Fig. 15(b).

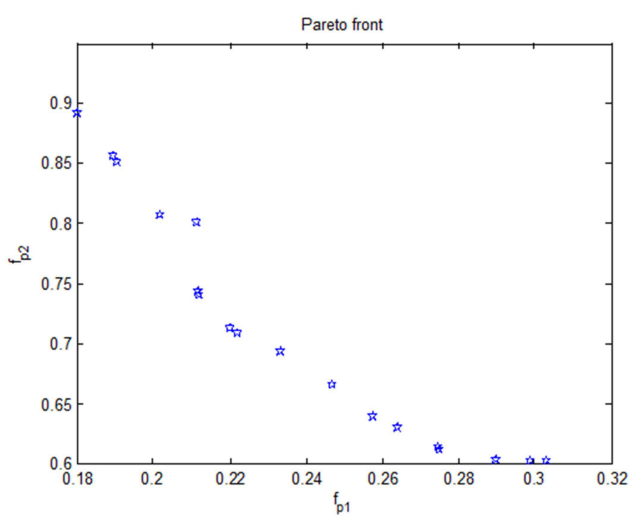

Fig. 14: Pareto optimal front

Table 2: Pareto front, criterion space

\begin{tabular}{llllllllll}
\hline & \multicolumn{8}{c}{ Solution No. } \\
$\beta_{\max }\left({ }^{\circ}\right)$ & 1 & 2 & 3 & 4 & 5 & 6 & 7 & 8 & 9 \\
$\delta$ & 30.2373 & 30.4556 & 30.1761 & 30.6109 & 30.5327 & 30.4886 & 30.5205 & 30.4490 & 30.6017 \\
$\Delta \phi\left(^{\circ}\right)$ & 0.8013 & 0.7444 & 0.7474 & 0.7209 & 0.7754 & 0.7912 & 0.7768 & 0.7928 & 0.7236 \\
\hline
\end{tabular}

Table 3: Pareto front, decision space

\begin{tabular}{|c|c|c|c|c|c|c|c|c|c|}
\hline \multirow[b]{3}{*}{$\lambda$} & \multicolumn{9}{|c|}{ Solution No. } \\
\hline & 1 & 2 & 3 & 4 & 5 & 6 & 7 & 8 & 9 \\
\hline & 0.5043 & 0.6293 & 0.6325 & 0.6636 & 0.5582 & 0.5269 & 0.5582 & 0.5237 & 0.6605 \\
\hline$S(\mathrm{~mm})$ & 116.9678 & 90.2519 & 81.1544 & 90.2491 & 116.9678 & 116.9706 & 116.9678 & 116.9706 & 90.2491 \\
\hline$\zeta\left({ }^{o}\right)$ & 12.9042 & 12.1974 & 12.1974 & 12.2011 & 12.9042 & 12.9042 & 12.4098 & 12.9042 & 12.2011 \\
\hline$M_{1}$ & 13 & 13 & 13 & 13 & 13 & 13 & 13 & 13 & 13 \\
\hline$M_{2}$ & 2 & 2 & 2 & 1 & 2 & 2 & 2 & 2 & 1 \\
\hline
\end{tabular}




\section{Conclusion}

In this study, micro machine tool oriented optimization of 3-RPS PKM is presented. A RPS-XY hybrid kinematic structure TRIPOD is proposed and designed for ultra-precision five-axis manufacturing. A newly represented orientation workspace is analyzed and calculated. The deflecting consistency of the stage in all directions, which determines the continuity and efficiency of manufacturing, is quantified by a new index. Moreover, structural and dimensional parameters of 3-RPS PKM are categorized as continuous and discrete, based on which their effects on rotational capacity are investigated. At last, by using a modified NSGA-II algorithm, an optimizer is established to design the stage.

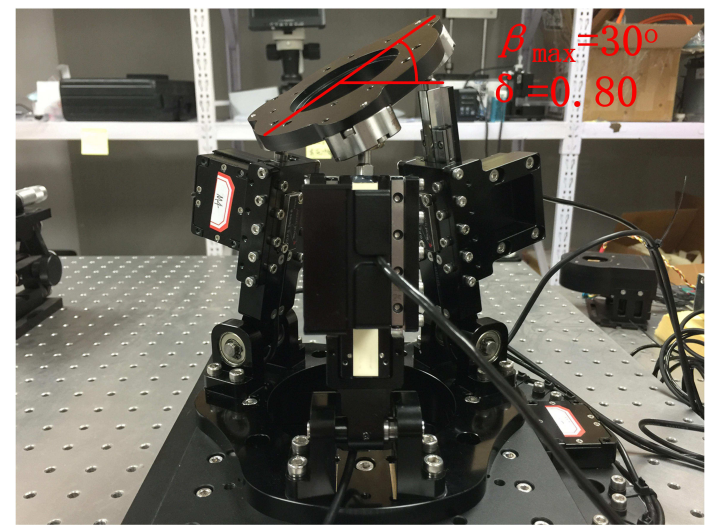

(a) The physical stage

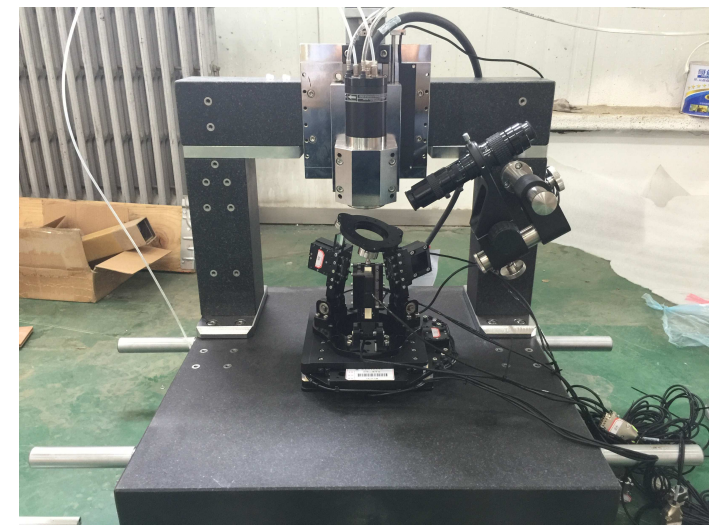

(b) The prototype of micro machine tool

Fig. 15: The physical stage and prototype of micro machine tool

\section{Acknowledgements}

The authors would like to acknowledge the support of National Natural Science Foundation of China. (Project No.: 51505020)

\section{Appendix A.}

The database of products of THK Cross Roller Guide (Table A1) and HEPHAIST SEIKO Spherical Rolling Joint (Table A2)

\section{References}

Chang, D. Y., Applications of the extent analysis method on fuzzy AHP, European journal of operational research, Vol.95, No.3 (1996), pp.649-655.

Deb, K., Pratap, A., Agarwal, S., and Meyarivan, T., A fast and elitist multiobjective genetic algorithm: NSGA-II, IEEE Transactions on Evolutionary Computation, Vol.6, No.2 (2002), pp.182-197.

Gosselin, C. M. and Guillot, M., The synthesis of manipulators with prescribed workspace, Journal of Mechanical Design, Vol.113, No.4 (1991), pp.451-455.

Hong, K. S. and Kim, J. G., Manipulability analysis of a parallel machine tool: application to optimal link length design, Journal of Robotic Systems, Vol.17, No.8 (2000), pp.403-415.

Huda, S. and Takeda, Y., Kinematic Analysis and Synthesis of a 3-URU Pure Rotational Parallel Mechanism with Respect to Singularity and Workspace, Journal of Advanced Mechanical Design Systems \& Manufacturing, Vol.1, No.1 (2007), pp.81-92.

Hunt K. H., Structural kinematics of in-parallel-actuated robot-arms, Journal of Mechanical Design, Vol.105, No.4 (1983), pp.705-712.

Kim, J., Park, C., Kim, J. and Park, F. C., Performance analysis of parallel mechanism architectures for CNC machining applications, Journal of manufacturing science and engineering, Vol.122, No.4 (2000), pp.753-759. 
Table A1: Linear guide model $M_{1}$

\begin{tabular}{|c|c|c|c|c|}
\hline$M_{1}$ & Product Model & $P(\mathrm{~mm})$ & $L_{0}(\mathrm{~mm})$ & $T(\mathrm{~mm})$ \\
\hline 0 & VR1-20×5Z & 12 & 20 & 1.6 \\
\hline 1 & VR2-30×5Z & 18 & 30 & 1.5 \\
\hline 2 & VR1-30×7Z & 22 & 30 & 1.6 \\
\hline 3 & VR1-40×10Z & 27 & 40 & 1.6 \\
\hline 4 & VR2-45×8Z & 24 & 45 & 1.5 \\
\hline 5 & VR1-50×13Z & 32 & 50 & 1.6 \\
\hline 6 & VR3-50×7Z & 28 & 50 & 2 \\
\hline 7 & VR2-60×11Z & 30 & 60 & 1.5 \\
\hline 8 & VR1-60×16Z & 37 & 60 & 1.6 \\
\hline 9 & VR1-70×19Z & 42 & 70 & 1.6 \\
\hline 10 & VR2-75×13Z & 44 & 75 & 1.5 \\
\hline 11 & VR3-75×10Z & 48 & 75 & 2 \\
\hline 12 & VR1-80×21Z & 52 & 80 & 1.6 \\
\hline 13 & VR4-80×7Z & 58 & 80 & 2 \\
\hline 14 & VR2-90×16Z & 50 & 90 & 1.5 \\
\hline 15 & VR3-100×14Z & 58 & 100 & 2 \\
\hline
\end{tabular}

Table A2: Spherical joint model $M_{2}$

\begin{tabular}{llll}
\hline$M_{2}$ & Product Model & $A, C, E(\mathrm{~mm})$ & $\left.\psi_{\max }{ }^{\circ}\right)$ \\
\hline 0 & SRJ006C & $25,5.5,11.5$ & 30 \\
\hline 1 & SRJ008C & $30,7,16$ & 30 \\
\hline 2 & SRJ012C & $42,11,20$ & 30 \\
\hline
\end{tabular}

Koenigsberger, F. and Tlusty, J., Machine tool structures (2013), Elsevier.

Lee K. M. and Shah D. K., Kinematic analysis of a three-degrees-of-freedom in-parallel actuated manipulator, Journal of Robotics and Automation, Vol.4, No.3 (1988), pp.354-360.

Li, Y. G., Liu, H. T., Zhao, X. M., Huang, T. and Chetwynd, D. G., Design of a 3-DOF PKM module for large structural component machining, Mechanism \& Machine Theory, Vol.45, No.6 (2010), pp.941-954.

Li, B., Yang, X., Hu, Y., Wang, Y. and Zhao, J., Dynamic Modeling and Design for the Parallel Mechanism of a Hybrid Type Parallel Kinematic Machine, Journal of Advanced Mechanical Design Systems \& Manufacturing, Vol.1, No.4 (2007), pp.481-492.

Liu, X. J., Tang, X., Wang, J., Huang, T. and Chetwynd, D. G., HANA: a novel spatial parallel manipulator with one rotational and two translational degrees of freedom, Robotica, Vol.23, No.2 (2005), pp.257-270.

Liu, Q. and Zhou, S., Hybrid genetic algorithm based on novel adaptive penalty function, Journal of Chongqing University (Natural Science Edition) (in Chinese), Vol.6, No.29 (2006), pp.78-81.

Shi, J., Wang Y. H., Zhang G., and Ding, H., Optimal design of 3-DOF PKM module for friction stir welding, International Journal of Advanced Manufacturing Technology, Vol.66, No.9-12 (2012), pp.1879-1889.

Shneor, Y. and Portman, V. T., Stiffness of 5-axis machines with serial, parallel, and hybrid kinematics: evaluation and comparison, CIRP Annals-Manufacturing Technology, Vol.59, No.1 (2010), pp.409-412.

Wang, Z., Wang, Z., Liu, W. and Lei, Y., A study on workspace, boundary workspace analysis and workpiece positioning for parallel machine tools, Mechanism \& Machine Theory, Vol.36, No.5 (2001), pp.605-622.

Whitley, D., A genetic algorithm tutorial, Statistics and computing, Vol.4, No.2 (1994), pp.65-85. 